\title{
INFLUENCE OF CBCT DIFFERENT VOXEL SIZES ON SOFT TISSUE THICKNESS MEASUREMENT
}

\author{
Raghdaa A. Mostafa*
}

\begin{abstract}
Objective: The aim of this study is to assess the effect of different CBCT voxel sizes on the soft tissue thickness measurements in a variety of maxillary and mandibular regions.

Methods: Two thicknesses (less than $2.0 \mathrm{~mm}$ and more than $2.0 \mathrm{~mm}$ ) of pink baseplate wax as simulated soft tissue were overlaid on a maxilla and mandible of a dry human skull. The thicknesses were measured with a digital caliper as the physical measurements. The skull was scanned using CBCT with four different voxel sizes $(0.20,0.25,0.30$ and $0.4 \mathrm{~mm})$. CBCT measurements were compared to the physical measurements. Intraclass Correlation Coefficient was used to assess both intra- and inter-observer reliability. Significance was considered to be $5 \%(\mathrm{P} \leq 0.05)$.
\end{abstract}

Results: Results showed a significant difference between the CBCT measurements using the 0.3 and $0.4 \mathrm{~mm}$ voxel sizes scans compared to the physical measurements when wax thickness was less than $2.0 \mathrm{~mm}$. On the other hand, no significant difference was found for the CBCT measurements using the four voxel sizes when thickness was greater than $2.0 \mathrm{~mm}$.

Conclusions: CBCT scans with voxel sizes of $0.3 \mathrm{~mm}$ and $0.4 \mathrm{~mm}$ provide accurate simulated soft tissue measurements when the thickness is more than $2.0 \mathrm{~mm}$. However, when greater visualization of details are required, scans with lower voxel sizes is required. Therefore, in a clinical situation, it is of great importance to customize the resolution protocol according to the accuracy needed.

KEYWORDS : Cone beam computed tomography; soft tissue; voxel size.

\section{INTRODUCTION}

The evaluation of soft tissue and its thickness is quite important in different disciplines of the dental field, such as during surgical correction after trauma or in case of anatomic, or developmental deformities, periodontal therapy, orthodontic treatment, and/ or restorative procedures ${ }^{[1]}$. Moreover, in dental implant treatment, the esthetic outcome depends on the accurate Gingival Biotype (GB) classification before implant surgery planning, especially in the anterior zone ${ }^{[2]}$. Therefore, knowing the condition of the peri-implant and periodontal tissue

\footnotetext{
* Oral and Maxillofacial Radiology, Faculty of Dentistry, Ain Shams University, Cairo, Egypt.
} 
preoperatively is important to measure the degree of risk in implant treatment ${ }^{[3]}$.

Several methods were used to evaluate the thickness of soft tissue. In the direct visual inspection, a probe is placed into the facial sulcus to confirm its transparency. This method is considered invasive and requires anesthesia. Special ultrasonic devices have been used but they have several drawbacks such as difficulty in maintaining transducer directionality, low availability, and high cost. Another common method but rather widely used is $\mathrm{CBCT}^{[2,3]}$.

CBCT has become a leading technology as an imaging modality in the medical diagnostic radiology with several advantages such as (i) the possibility to acquire images with subjects in the upright position; (ii) providing the radiologists with high-resolution images while using less ionizing radiation for the patient compared to multislice $\mathrm{CT}$, (iii) clinically acceptable, and (iv) high precision regarding all the linear, angular and volumetric measurements of hard tissue with an accuracy of $1.0 \mathrm{~mm}^{[1,4,5]}$.

However, various limitations are present in CBCT including poor soft tissue contrast. This results in the inability of $\mathrm{CBCT}$ to distinguish between tissue attenuation differences. This limited contrast resolution remains a barrier to the extension of CBCT technologies into diagnostic imaging, in which the detection of small changes in soft-tissue attenuation is a priority ${ }^{[6]}$.

Several parameters are commonly enlisted to influence the image quality such as voxel size, the field of view (FOV), tube voltage, tube current and the type of CBCT device. Therefore, different imaging protocols are available allowing for variations in voxel sizes variation to adapt the image resolution to the specific diagnostic task ${ }^{[7,8]}$.

Various attempts have been made to evaluate the effect of changing the voxel size on image quality in different dental applications including external root fracture ${ }^{[8]}$, detection of artificially induced recurrent caries-like lesions ${ }^{[9]}$, periodontal defects ${ }^{[10]}$, and volumetric measurements of the mandible ${ }^{[11]}$.

Studies have also been conducted to measure soft tissue thickness in patients using CBCT. In the orthodontic practice, palatal soft tissue thickness was assessed at popular placement sites of temporary anchorage devices ${ }^{[12]}$. In forensic medicine, the reliability of facial soft tissue thickness obtained by CBCT was evaluated for craniofacial reconstruction ${ }^{[13,14]}$. Moreover, soft tissue thickness was investigated prior to the planning of esthetic crown lengthening procedures and for evaluating periodontal soft tissues ${ }^{[15]}$. One study investigated the accuracy of CBCT in measuring soft tissue thickness using a dry skull with different pink baseplate wax thickness. This study did not address the effect of different voxel sizes of the CBCT on the accuracy of measurements ${ }^{[1]}$.

To date, the influence of voxel size on the accuracy of measuring soft tissue thickness based on comparative measurements of physical objects was not investigated. Therefore, the aim of this study was to investigate and compare the accuracy of CBCT in measuring soft tissue thickness at different locations using four voxel sizes and the reference physical measurements obtained by a digital caliper

\section{MATERIALS AND METHODS}

The present study was approved by the Human Research Ethics Committee of the Faculty of Dentistry, Ain Shams University, Cairo, Egypt. One dry skull was obtained from the Department of Anatomy, Faculty of Medicine, Ain Shams University, Cairo, Egypt. The dry skull had no history or background.

A layer of pink baseplate wax was used as a soft tissue simulation. The wax was placed on the maxilla and mandible of the dry skull. Careful consideration was taken to ensure the wax was applied uniformly 
and bubbles free. The wax was covering the buccal and palatal cortical bones of the maxilla, as well as the buccal, lingual cortical bones, and crestal bone of the mandible. An endodontic spreader (size \#35) with a stopper was used to pierce the wax until the surface of the bone was reached. This step was done to create holes perpendicular to the wax surface in different regions in both the maxilla and mandible.

A total of 32 different locations were selected. Sixteen (16) holes at the mandible were located at the superior one-third of the height of the mandible. Six (6) holes were located in the anterior area at the buccal and lingual cortical plates at the center and at the canine regions bilaterally. Five (5) holes were located in the posterior area bilaterally. Four (4) holes were located in the buccal and lingual cortical plates at the premolar and molar regions and an additional one (1) hole was located at the crestal bone at the molar area.

The other 16 holes were located in the maxilla. Six (6) holes at the buccal and palatal cortical plates at the center and at the canine regions bilaterally. Eight (8) holes were located in the posterior area bilaterally. Four (4) holes were located in the buccal and palatal cortical plates at the premolar and molar regions. Additional two (2) holes were located in the middle and posterior over the midpalatal suture.

The stopper was placed parallel to the wax surface and the length of the spreader till the stopper was measured using a digital caliper to present the physical measured thickness of the pink baseplate wax. The physical measurements were repeated twice with one-week interval. The average value of the measurements was considered as the gold standard or real measurement. A small piece of gutta-percha was placed inside the hole created by the spreader as a reference point and guide for the path of measurement.

The skull was scanned using i-CAT Next Generation (i-CAT; Imaging Sciences International, Hatfield, PA) present at the Faculty of Dentistry,
Ain-shams University. The indicating lights were adjusted in accordance with the manufacturer's instructions and recommendations. A cotton roll was placed between the arches for better stabilization of the skull. The images were acquired with the i-CAT vision software on a computer with the following settings: FOV (field of view) of $8 \mathrm{~cm} \times 11 \mathrm{~cm}$; voxel size: $0.20 \mathrm{~mm}$; focal spot: $0.50 \mathrm{~mm} ; 120 \mathrm{kVp}$; image detector: amorphous silicon flat panel; and image acquisition: single $360^{\circ}$ rotation. Following the first CBCT scan, three more scans of the same regions were performed using the same parameters but with three different voxel sizes $0.25 \mathrm{~mm}, 0.30$ $\mathrm{mm}$ and $0.40 \mathrm{~mm}$.

The images were transformed to Digital Imaging and Communications in Medicine (DICOM) and then the i-CAT vision commercial software was used to perform the soft tissue thickness measurements. The software features such as magnification, contrast, and brightness changes were used to ensure accurate measurements.

\section{Image Analysis}

A total of 32 areas were investigated and each area was analyzed individually. Using the multiplanar reconstruction (MPR) screen, the axial images with a slice thickness of $0.10 \mathrm{~mm}$ were selected and the gutta percha in each location was identified by scrolling through sequential slices. The axial slice which clearly identifies and represents the maximum mesiodistal dimension of the gutta percha in each location was selected. On the selected slice, the sagittal plane passes antero-posteriorly along the gutta percha. The coronal plane was adjusted to be passing perpendicular to the sagittal plane and through the middle of the gutta percha as shown in (Fig .1 a). On the sagittal image, the axial plane was adjusted to be passing perpendicular to the coronal plane and parallel to the full length of the gutta percha.

Using this non-orthogonal sagittal image and after slice preparation, the soft tissue thickness was measured just above a line that is parallel to the 


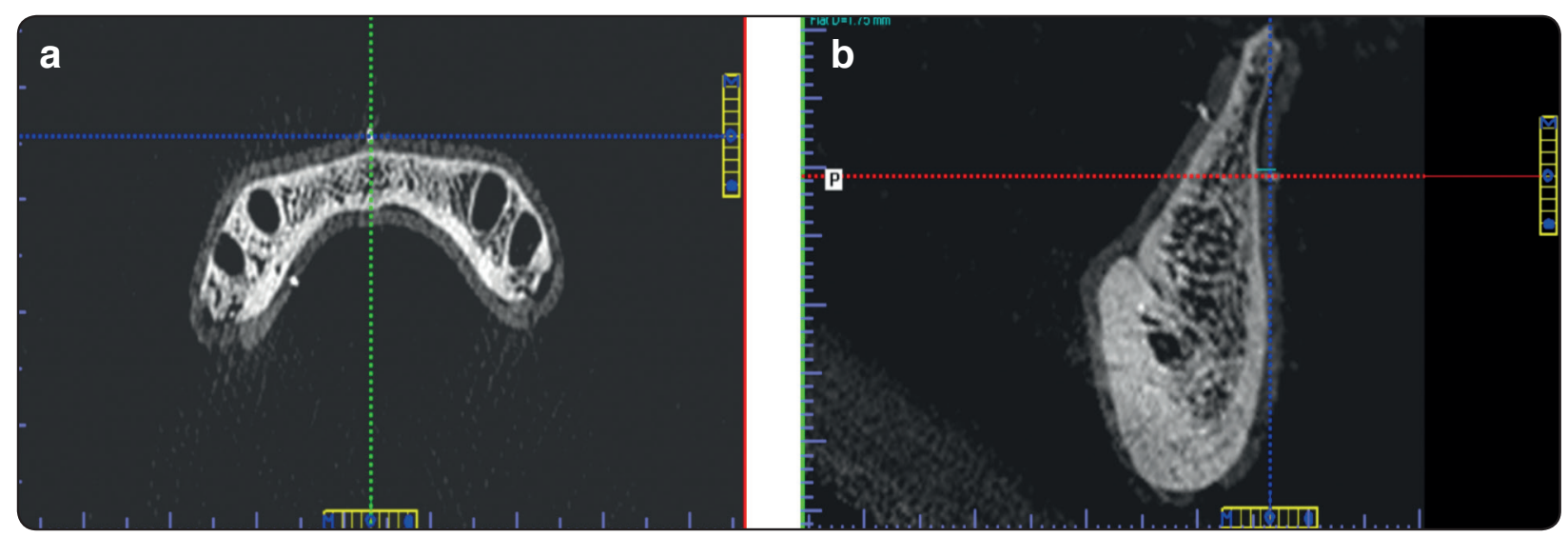

Fig. (1) Measurement of pink baseplate wax thickness in the buccal region of anterior mandible. (a) Axial image, (b) Non-orthogonal sagittal image showing the measurement $(1.75 \mathrm{~mm})$

gutta percha as shown in (Fig. $1 \mathrm{~b}$ ). The software automatically displayed the thickness measurements in millimeters, which were recorded for further analysis. These steps were repeated for each region with a total number of 16 measurements in the mandible and 16 measurements in the maxilla.

The above steps were repeated after applying another layer of pink baseplate wax on the previous layer. The new layer was gently pressed together to avoid creating any voids between the layers. Physical measurements were recorded in the same way. CBCT scans with the four different voxel sizes $(0.20,0.25,0.30$ and $0.40 \mathrm{~mm})$ were performed.

All measurements were analyzed two times with the i-CAT vision software by an experienced oral and maxillofacial radiologist with more than 10 years of experience in this field. The measurements took place in two-weeks intervals to evaluate the intra-observer reproducibility.

Another oral and maxillofacial radiologist followed the same measurement protocol to evaluate the inter-observer reproducibility.

\section{Statistical Analysis}

Statistical evaluation was performed using SPSS 23.0 (SPSS Inc, Chicago, IL). The mean and standard deviation of the measurements for all different scans were calculated. To calculate the intra-observer and inter-observer reliability of CBCT measurements, an Intraclass Correlation Coefficient (ICC) and one sample t-test were used. Repeated measures (ANOVA) analysis of variance followed by posthoc Bonferroni test was performed to compare the physical and CBCT measurements. Results with $\mathrm{P}<$ 0.05 were considered statistically significant.

\section{RESULTS}

Intraclass correlation coefficient (ICC) analysis showed that all intra- observer reliability coefficients obtained from the CBCT measurements for the four voxel sizes were greater than 0.7 , which indicates high reproducibility for the CBCT measurements.

Table 1 shows the results of ICC analysis and t-test for the inter-observer reproducibility. There were no significant differences between the two observers for CBCT measurements. The results of ICC analysis for all measurements were $>0.70$, which indicates high inter-examiner assessment reliability.

Tables 2 and 3 present measurements in different areas, methods of measurement, the mean of the CBCT measurements for the different voxel sizes, standard deviation (SD), mean differences between the CBCT measurement and the physical 
measurement and the P-value when one layer of pink bases wax was overlaid. Table 3 presents the same data when two layers of pink baseplate wax were overlaid

In post hoc comparisons; when one layer of pink wax was used (mean thickness of $1.4 \mathrm{~mm}$ ), both the physical and CBCT measurements showed no significant difference in the anterior and posterior regions of the jaws when using voxel sizes of 0.2 $\mathrm{mm}$ and $0.25 \mathrm{~mm}$. On the other hand, a significant difference in both the anterior and posterior areas was found when voxel sizes of $0.30 \mathrm{~mm}$ and 0.40 mm were used $(P<0.05)$ and as shown in Table 2.

No significant differences were found between the physical measurements obtained from the digital caliper and the CBCT measurements in the anterior and posterior regions of the jaws for the four voxel sizes used in the study when two layers of pink base wax were overlaid (thickness $>2.0 \mathrm{~mm})(P>0.05)$ as shown in Table 3 .

TABLE (1): Inter-observer reproducibility at different voxel sizes

\begin{tabular}{|c|c|c|c|c|c|c|}
\hline Pink base wax & & Examiner 1 & Examiner 2 & Difference & Significant by t-test & ICC \\
\hline \multirow{2}{*}{$\begin{array}{c}\text { One layer } \\
\text { Th }=1.4 \mathrm{~mm}\end{array}$} & 0.20 voxel & $1.37 \pm 0.19$ & $1.36 \pm 0.19$ & 0.01 & 0.80 & 0.87 \\
\cline { 2 - 7 } & 0.25 voxel & $1.32 \pm 0.23$ & $1.30 \pm 0.21$ & 0.02 & 0.59 & 0.81 \\
\cline { 2 - 7 } & 0.30 voxel & $1.31 \pm 0.23$ & $1.31 \pm 0.20$ & 0.00 & 0.96 & 0.76 \\
\cline { 2 - 7 } & 0.40 voxel & $1.20 \pm 0.24$ & $1.26 \pm 0.14$ & -0.06 & 0.05 & 0.77 \\
\hline \multirow{4}{*}{$\begin{array}{c}\text { Two layers } \\
\text { Th }>2.0 \mathrm{~mm}\end{array}$} & 0.20 voxel & $2.63 \pm 0.46$ & $2.62 \pm 0.45$ & 0.020 & 0.57 & 0.95 \\
\cline { 2 - 7 } & 0.25 voxel & $2.67 \pm 0.45$ & $2.69 \pm 0.49$ & -0.02 & 0.72 & 0.91 \\
\cline { 2 - 7 } & 0.30 voxel & $2.53 \pm 0.43$ & $2.59 \pm 0.46$ & -0.06 & 0.21 & 0.95 \\
\cline { 2 - 7 } & 0.40 voxel & $2.53 \pm 0.42$ & $2.56 \pm 0.41$ & -0.03 & 0.42 & 0.93 \\
\hline
\end{tabular}

Th (Thickness)

TABLE (2) Comparison of measurements for one layer of pink baseplate wax

\begin{tabular}{|c|c|c|c|c|c|}
\hline Area & Method & Mean (mm) & SD & Mean differences $(\mathrm{mm})$ & $\mathrm{P} *$ value \\
\hline \multirow{5}{*}{ Anterior area } & Physical measurement & 1.41 & 0.24 & & \\
\hline & 0.20 voxel & 1.33 & 0.22 & 0.08 & NS \\
\hline & 0.25 voxel & 1.29 & 0.18 & 0.13 & NS \\
\hline & 0.30 voxel & 1.23 & 0.17 & 0.179 & $0.03 *$ \\
\hline & 0.40 voxel & 1.12 & 0.15 & 0.294 & $0.00 *$ \\
\hline \multirow{5}{*}{ Posterior area } & Physical measurement & 1.49 & 0.19 & & \\
\hline & 0.20 voxel & 1.38 & 0.17 & 0.10 & NS \\
\hline & 0.25 voxel & 1.36 & 0.21 & 0.13 & NS \\
\hline & 0.30 voxel & 1.30 & 0.22 & 0.191 & $0.00 *$ \\
\hline & 0.40 voxel & 1.26 & 0.17 & 0.226 & $0.00 *$ \\
\hline
\end{tabular}


TABLE (3) Comparison of measurements for two layers of pink baseplate wax

\begin{tabular}{|c|c|c|c|c|c|}
\hline Area & Method of measurement & Mean $(\mathrm{mm})$ & SD & Mean differences $(\mathrm{mm})$ & $\mathrm{P}^{*}$ value \\
\hline \multirow{5}{*}{ 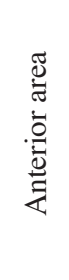 } & Physical measurement & 2.59 & 0.40 & & \\
\hline & 0.20 voxel & 2.69 & 0.39 & -0.10 & NS \\
\hline & 0.25 voxel & 2.65 & 0.43 & -0.06 & NS \\
\hline & 0.30 voxel & 2.55 & 0.38 & 0.04 & NS \\
\hline & 0.40 voxel & 2.54 & 0.42 & 0.05 & NS \\
\hline \multirow{5}{*}{ 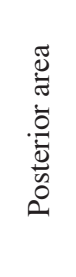 } & Physical measurement & 2.55 & 0.49 & & \\
\hline & 0.20 voxel & 2.60 & 0.46 & -0.05 & NS \\
\hline & 0.25 voxel & 2.65 & 0.43 & -0.10 & NS \\
\hline & 0.30 voxel & 2.51 & 0.43 & 0.04 & NS \\
\hline & 0.40 voxel & 2.54 & 0.43 & 0.01 & NS \\
\hline
\end{tabular}

$* P<0.05$ considers statistically significant

\section{DISCUSSION}

It is very vital to consider the influence of the inherent image quality on the reliability and accuracy of the diagnostic outcome. An inverse relationship was found between voxel size and image quality. Although images with small voxel sizes might appear sharper from a subjective point of view, this comes with the expense of increasing the radiation dose to the patients and might provide the same diagnostic outcome as lower resolution images. Therefore, it is critical to compare CBCT examinations using various voxel settings in different dental applications ${ }^{[16]}$.

The CBCT scan parameters selection for various diagnostic tasks, voxel resolution included, have been performed almost arbitrarily depending on the availability in the device setting ${ }^{[16]}$. In an attempt to use the best setting to accurately detect simulated soft tissue thickness, this study was conducted to examine the results of four voxel sizes available in the FOV of $8 \mathrm{~cm} \times 11 \mathrm{~cm}$ in the i-CAT ${ }^{\circledR N} \mathrm{Next}$ Generation CBCT machine in comparison with physical measurements.

Younes et al. ${ }^{[17]}$ investigated both the buccal bone and gingival thickness and stated that the mean

\section{NS: Not significant}

gingival thickness at central incisors was found to be $1.37 \mathrm{~mm}, 1.33 \mathrm{~mm}$ at lateral incisors, and 1.08 $\mathrm{mm}$ at canines. Another investigation by La Rocca et al. ${ }^{[18]}$, revealed that the mean crestal gingival thickness was found to be $1.09 \mathrm{~mm}$. Based on that, it is important to recognize the ability of CBCT to accurately detect small soft tissue thickness less than $2.0 \mathrm{~mm}$. Pink baseplate wax was used in this study as a soft tissue simulation and overlaid on the dry skull. Pink wax is accepted in the literature and was used by several studies to simulate soft tissue in patients imaging ${ }^{[8,11]}$.

In the present study, MPR was used for better standardization of the exact orientation of the direction of the hole in the pink baseplate wax. The results of the intraclass correlation coefficient analysis showed high inter and intra- observer reproducibility in all the readings.

Using MPR is common and has been used to measure the wax thickness in this study. De Donno et al, ${ }^{[4]}$ used the MPR to measure 12 easily recognizable bone landmarks, corresponding to well-defined anatomic structures, with an acceptable approximation of the underlying bone surface. Bulut et al, ${ }^{[8]}$ used MPR and sagittal images along 
each tooth plane to measure the diameter and depth of the external root resorptions cavities.

A marked clarity and ease of identification of the soft tissue were noted with $0.20 \mathrm{~mm}$ voxel size images compared to larger voxel sizes especially when the thickness was less than $2.0 \mathrm{~mm}$, but the subjectivity in image evaluation was not the aim of this study.

The results of this study found that measurements using the four voxel sizes were comparable with no significant difference when the thickness was more than $2.0 \mathrm{~mm}$. Our results were consistent with the investigation done by Meundi and David ${ }^{[5]}$. Authors reported that, in the craniofacial reconstruction, voxel sizes of 0.30 and $0.40 \mathrm{~mm}$ were better used to evaluate facial soft tissue thickness. The authors stated that using a smaller voxel size of $0.20 \mathrm{~mm}$ would result in a very large surface mesh model, which makes it difficult to process an accurate 3D surface model. Furthermore, the authors confirmed that using smaller voxel size would increase the radiation dose received by the patient. Note that Meundi and David ${ }^{[5]}$ did not consider the accuracy of the soft tissue thickness against gold standard but rather evaluated the sex and racial differences in the soft tissue values.

A study by Moudi et al, ${ }^{[1]}$ in which different thicknesses of pink baseplate wax were applied on a dry human skull. The skull was scanned by using two CBCT scanners with 0.01 and $0.15 \mathrm{~mm}$ voxel sizes. Results showed no significant difference between CBCT and digital caliper measurements in thickness less than $2.0 \mathrm{~mm}$, while a significant difference was observed for thicknesses greater than $2.0 \mathrm{~mm}(\mathrm{P}<0.05)$. In contrast, in the present study, the increased voxel size showed significant results when the thickness is less than $2.0 \mathrm{~mm}$. However, there was no significant difference between the voxel sizes when the thickness was greater than $2.0 \mathrm{~mm}$. Differences between the results of the studies may be due to differences in observer performance, the device settings, or the software used. It is important to mention that the effect of different voxel sizes was not investigated by Moudi et al ${ }^{[1]}$

Fokas et al, $2018^{[19]}$ stated that the range of differences between CBCT and "gold standard" measurements which often exceeded the $1.0 \mathrm{~mm}$ threshold, were not statistically significant. This was in agreement with the results of this study, as the mean difference ranged from $(0.08-0.29 \mathrm{~mm})$. The results were not significant for all voxel sizes when the thickness was more than $2.0 \mathrm{~mm}$.

In the present study, there was a tendency to underestimate the linear measurements on CBCT images when compared with the physical measurement especially when the pink baseplate wax thickness was less than $2.0 \mathrm{~mm}$. A systematic review on the accuracy of linear measurement in CBCT images found a wide range of error with no clear trends as to whether measurements are consistently under-or overestimated in comparison with a gold standard ${ }^{[19]}$.

Many studies evaluated the effect of voxel sizes on the accuracy of various dental situations. Torres et al, ${ }^{[20]}$ used (i-CAT device ${ }^{\circledR}$ ) with four different voxel sizes protocols $(0.20,0.25,0.30$, and 0.40 $\mathrm{mm}$ voxels,) to evaluate the accuracy of linear measurements on eight dry mandibles. The authors reported that the four protocols were comparable and the majority of the values were underestimating the real values, which is in agreement with the present results. In addition, the authors stated that the use of smaller voxels did not influence the accuracy of measurement although the evaluations with smaller voxels were easier ${ }^{[20]}$. The outcome of this study is in good agreement with the results of the current investigation.

Several studies reported that smaller voxel sizes did not lead to greater accuracy; Sang et al, ${ }^{[21]}$ found that decreasing voxel size from 0.30 to $0.15 \mathrm{~mm}$ did not result in increased accuracy of $3 \mathrm{D}$ tooth reconstruction. This is in agreement with other CBCT studies comparing different voxel 
sizes $(0.075,0.10,0.15$, and $0.20 \mathrm{~mm})$ in terms of determining the depth and diameter of the external root resorption on the apical and cervical regions ${ }^{[22]}$. Fokas et al, ${ }^{[19]}$ concluded that for implant treatment planning, voxel sizes of 0.30 to $0.40 \mathrm{~mm}$ were adequate to provide CBCT images of acceptable diagnostic quality. The authors' conclusions are also in agreement with our results where the difference in measurements using the selected four voxel sizes was insignificant when the thickness of the soft tissues is more than $2.0 \mathrm{~mm}$.

On the other hand, high resolution has been reported to influence diagnostic quality in other fields such as the detection of periodontal defects ${ }^{[10]}$ and artificially induced recurrent caries-like lesions [9]. As shown in this study, voxel sizes of 0.20 and $0.25 \mathrm{~mm}$ provide better accuracy when the soft tissues thickness is less than $2.0 \mathrm{~mm}$.

\section{CONCLUSION}

The current study addresses the comparison made between measured soft tissue thicknesses using CBCT four voxel sizes and the gold standard measurements in different maxillary and mandibular regions. CBCT scans with voxel sizes of 0.30 and 0.40 provide highly accurate and reproducible simulated soft tissue measurements when the thickness is more than $2.0 \mathrm{~mm}$. However, when greater visualization of details is required, as in case of soft tissue thickness less than $2.0 \mathrm{~mm}$, scans with lower voxel sizes $(0.2$ and $0.25 \mathrm{~mm}$ in comparison with 0.3 and $0.4 \mathrm{~mm}$ ) are recommended. Therefore, in a clinical situation, it is of great importance to customize the resolution protocol according to the intended diagnostic purpose of each scan.

Funding: The author(s) received no financial support for the research, authorship, and/or publication of this article.

Declaration of Conflicting Interests: The author(s) declared no potential conflicts of interest with respect to the research, authorship, and/or publication of this article

\section{REFERENCES}

1. Moudi E, Haghanifar S, Johari M, Gholinia H, Ghanbarabadi MK. Evaluation of the cone-beam computed tomography accuracy in measuring soft tissue thickness in different areas of the jaws. J Indian Soc Periodontol. 2019;23:334-338

2. Wang L, Chen J, Zhang W, Wu H, Yang F. Accuracy of assessing gingival thickness in the esthetic maxillary region by periodontal probing, cone-beam computed tomography and digital scanning. Int J Clin Exp Med. 2019;12(8):10302-10309.

3. Kobayashi T, Nakano T, Ono S, Matsumura A, Yamada S, Yatani H. Quantitative evaluation of connective tissue grafts on peri-implant tissue morphology in the esthetic zone: A 1-year prospective clinical study. Clin Implant Dent Relat Res. 2020;(9):1-8.

4. De Donno A, Sablone S, Lauretti C, Mele F, Martini A, Introna $\mathrm{F}$, et al., Facial approximation: Soft tissue thickness values for Caucasian males using cone beam computer tomography. Leg Med. 2019;37(1):49-53.

5. Meundi MA, and David CM. Application of cone beam computed tomography in facial soft tissue thickness measurements for craniofacial reconstruction. J Oral Maxillofac Pathol., 2019; 23:114-121.

6. Miracle AC, and Mukherji SK. Cone beam CT of the Head and Neck, Part 1: Physical Principles. Ajnr. Am. J. Neuroradiol. 2009; 30 (6) 1088-95.

7. Lindfors $\mathrm{N}$, Lund H, Johansson H, Ekestubbe A. Influence of patient position and other inherent factors on image quality in two different cone beam computed tomography (CBCT) devices. Eur J Radiol Open. 2017(11)1; 4:132-137.

8. Goller Bulut D, Uğur Aydın Z. The impact of different voxels and exposure parameters of $\mathrm{CBCT}$ for the assessment of external root resorptions: A phantom study. Aust Endod J. 2019;45(2):146-153.

9. Sousa Melo SL, Belem MDF, Prieto LT, Tabchoury CPM, Haiter-Neto F. Comparison of cone beam computed tomography and digital intraoral radiography performance in the detection of artificially induced recurrent carieslike lesions. Oral Surg Oral Med Oral Pathol Oral Radio. 2017(9);124(3):306-314.

10. Kolsuz ME, Bagis N, Orhan K, Avsever H, Demiralp KO. Comparison of the influence of FOV sizes and different 
voxel resolutions for the assessment of periodontal defects. Dentomaxillofac Radiol 2015; 44(7): 20150070.

11. Dong T, Xia L, Cai C, Yuan L, Ye N, Fang B. Accuracy of in vitro mandibular volumetric measurements from $\mathrm{CBCT}$ of different voxel sizes with different segmentation threshold settings. BMC Oral Health. 2019;19:206-212

12. Vu T, Bayome M, Ah Kook Y, Ho Han s. Evaluation of the palatal soft tissue thickness by cone-beam computed tomography. Korean J Orthod. 2012 (12); 42(6): 291-296.

13. Hwang HS, Choe SY, Hwang JS, Moon DN, Hou Y, Lee WJ, et al. Reproducibility of facial soft tissue thickness measurements using Cone-Beam CT images according to the measurement methods. J Forensic Sci. 2015 (7);60(4):957-965.

14. Cassiano LS, Barriviera M, Suzuki S, Nascimento GG, Januario AL, Hilgert LA, et al, Soft tissue cone beam computed tomography (ST-CBCT) for the planning of esthetic crown lengthening procedures. Int $\mathrm{J}$ Esthet Dent. 2016;11(4):482-493.

15. Panda M, Jalaluddin MD, Agrawal U. Soft tissue-Cone Beam Computed Tomography: A clinico radiographic study. Acta Scientific Dental Sciences 3.5(2019): 02-07.

16. Spin-Neto R, Gotfredsen E, Wenzel A. Impact of voxel size variation on CBCT-based diagnostic outcome in dentistry: a Systematic Review. J Digit Imaging.2013 26:813-820
17. Younes F, Eghbali A, Raes M, De Bruyckere T, Cosyn J, De Bruyn H. Relationship between buccal bone and gingival thickness revisited using non-invasive registration methods. Clin Oral Implants Res. 2016 (5); 27(5):523-528

18. La Rocca AP, Alemany AS, Levi P Jr, Juan MV, Molina JN, Weisgold AS. Anterior maxillary and mandibular biotype: relationship between gingival thickness and width with respect to underlying bone thickness. Implant Dent. 2012 (12); 21(6):507-515

19. Fokas G, Vaughn VM, Scarfe WC, Bornstein MM. Accuracy of linear measurements on CBCT images related to presurgical implant treatment planning: A systematic review. Clin Oral Implants Res. 2018 (10);29 393-415

20. Torres MG, Campos PS, Segundo NP, Navarro M, CrusoéRebello I. Accuracy of linear measurements in cone beam computed tomography with different voxel sizes. Implant Dent. 2012(4);21(2):150-155.

21. Sang YH, Hu HC, Lu SH, Wu YW, Li WR, Tang ZH. Accuracy assessment of three-dimensional surface reconstructions of in vivo teeth from cone-beam computed tomography. Chin Med J. 2016;129(12):1464-1470.

22. Sonmez G, Koc C, Kamburoglu K. Accuracy of linear and volumetric measurements of artificial ERR cavities by using CBCT images obtained at 4 different voxel sizes and measured by using 4 different software: an ex vivo research. Dentomaxillofac Radiol 2018; 20170325. 